\title{
Complement Factor 3 Could Be an Independent Risk Factor for Mortality in Patients with HBV Related Acute-on-Chronic Liver Failure
}

\author{
Geng-lin Zhang, ${ }^{1,2}$ Ting Zhang, ${ }^{3}$ Yi-nong Ye, ${ }^{4}$ Jing Liu, ${ }^{1,2}$ Xiao-hong Zhang, ${ }^{1,2}$ Chan Xie, ${ }^{1,2}$ \\ Liang Peng, ${ }^{1,2}$ and Zhi-liang Gao ${ }^{1,2,5}$ \\ ${ }^{1}$ Department of Infectious Diseases, The Third Affiliated Hospital of Sun Yat-Sen University, Guangzhou 510630, China \\ ${ }^{2}$ Guangdong Provincial Key Laboratory of Liver Disease, The Third Affiliated Hospital of Sun Yat-Sen University, \\ Guangzhou 510630, China \\ ${ }^{3}$ Department of Ultrasound, The Third Affiliated Hospital of Sun Yat-Sen University, Guangzhou 510630, China \\ ${ }^{4}$ Department of Infectious Diseases, Foshan Hospital of Sun Yat-Sen University, Foshan 528000, China \\ ${ }^{5}$ Key Laboratory of Tropical Disease Control, Sun Yat-Sen University, Ministry of Education, Guangzhou 510630, China
}

Correspondence should be addressed to Liang Peng; pzp33@hotmail.com and Zhi-liang Gao; zhilianggao@21cn.com

Received 3 December 2015; Revised 8 March 2016; Accepted 20 March 2016

Academic Editor: Paul M. Tulkens

Copyright (C) 2016 Geng-lin Zhang et al. This is an open access article distributed under the Creative Commons Attribution License, which permits unrestricted use, distribution, and reproduction in any medium, provided the original work is properly cited.

\begin{abstract}
The complement is thought to be involved in the pathogenesis of multiple liver disorders. However, its role in patients with HBV related acute-on-chronic liver failure (HBV-ACLF) remains unclear. Serum levels of the third and fourth complement components $(\mathrm{C} 3, \mathrm{C} 4)$ and complement function (CH50) were examined in this prospective, observational study. Associations between their expression and disease activity were analyzed. Survival was analyzed by Kaplan-Meier curves. Predictors of clinical outcome were determined by Cox regression analysis. C3, C4, and CH50 levels were significantly lower in HBV-ACLF patients compared to controls. C3, C4, and CH50 levels were negatively correlated with Tbil levels but positively associated with PTA levels. C3 levels were negatively associated with MELD-Na. C3 levels were significantly lower in HBV-ACLF patients who died compared to patients who survived. In a median hospital stay of 39 days, mortality occurred in 41 patients with a progressive increase based on C3 grade $(P=0.008)$. The actuarial probability of developing mortality was significantly higher in patients with low $\mathrm{C} 3$ grade compared to those with high C3 grade $(P<0.001)$. Multivariate Cox regression analysis showed that C3 levels were an independent predictor of mortality. Complement played a pathogenic role in HBV-ACLF patients and C3 was an independent predictor of mortality.
\end{abstract}

\section{Introduction}

Chronic hepatitis $\mathrm{B}(\mathrm{CHB})$ resulting from a variety of hepatic disease processes caused by HBV infection can lead to acuteon-chronic liver failure (HBV-ACLF), which is a severe clinical syndrome characterized by an acute deterioration of liver function with the eventual development of multiple organ failure $[1,2]$. A poor understanding of the pathogenesis of HBV-ACLF and lack of effective treatment options result in extremely high mortality rates $[1,2]$. There is a growing appreciation that immunity-mediated inflammation plays an important role in the pathogenesis of HBV-ACLF [3]. In particular, different arms of the innate and adaptive immune system make critical contributions to the progression of
HBV-ACLF [1]. However, it is not clear if the complement, which is an important bridge between the innate and adaptive immune systems, plays a role in the pathogenesis of HBVACLF.

The complement system comprises approximately 30 proteins that are present either as soluble factors or as membrane-associated proteins [4]. The complement can be activated via the classical, lectin, or alternative pathways, resulting in $\mathrm{C} 3$ activation and leading to the generation of the membrane attack complex (C5b-9). Complement activation has also been reported to activate multiple immune cells and play an important role in host defense and wound healing by increasing secretion of inflammatory cytokines. However, 
overactivation of complement components can lead to tissue necrosis and multiorgan dysfunction [5-7].

The role of the complement has been implicated in liver regeneration after partial hepatectomy [8], liver fibrosis [9], ischemia reperfusion [10], alcoholic liver disease [11], nonalcoholic steatohepatitis [12], and viral persistence in patients with $\mathrm{CHB}$ or chronic hepatitis $\mathrm{C}$ infections $[4,13]$. However, there are few studies which investigated its role in patients with HBV-ACLF. An animal liver failure model recently showed activation of complement as evidenced by the hepatic deposition of C3 and C5b-9. Compared with wild-type mice, C3-/- mice survived significantly longer and displayed reduced liver inflammation [14]. Another study using liver specimens from two patients with HBV-ACLF revealed a significant deposition of complement components in the liver parenchyma [15]. Given the observation that complement may be activated in patients with HBV-ACLF, we sought to determine the role of complement in a large sample cohort of Chinese patients with HBV-ACLF and to explore its relationship with disease activity. We tested the hypothesis that complement may play a role in the pathogenesis of HBVACLF and could be an independent risk factor for mortality in $\mathrm{HBV}$-ACLF patients.

\section{Patients and Methods}

2.1. Study Design and Patients. In this prospective, observational study, we enrolled HBV-ACLF patients who were admitted to our department between April 2009 and March 2010. Adult HBV-ACLF patients who were willing to participate in and consented to the study were screened for this study based on previously described criteria [16]. Among exclusion criteria were (1) presence of other liver diseases including autoimmune liver diseases, Wilson's disease, or evidence of cancer and liver cirrhosis; (2) coinfection with hepatitis A, C, D, or E or HIV virus; (3) treatment with artificial liver support or immunomodulatory drugs within 6 months prior to the screening or during the hospital stay; (4) history of drug abuse or alcohol abuse; and (5) history of renal, cardiovascular, pulmonary, endocrine, or rheumatic diseases. Pregnant women were excluded. Cirrhosis was diagnosed when a small, nodular liver was seen on ultrasound, computerized tomography scans, or magnetic resonance, with the exclusion of primary biliary cirrhosis and cirrhosis caused by schistosome [17]. Clinical assessment and blood samplings were performed at admission and prior to initiation of treatment. Each patient was treated with the same comprehensive supportive treatment (i.e., reduced glutathione, glycyrrhizin, ademetionine, polyene phosphatidylcholine, alprostadil, plasma or albumin transfusion if needed, and antiviral therapy using nucleos(t)ide analogues if HBV-DNA was detected.) [17]. The endpoint of this study was mortality when patients were discharged from our department. We also enrolled $35 \mathrm{CHB}$ patients and 16 healthy controls (NC) from our hospital. CHB were diagnosed according to previously described criteria [16]. Peripheral blood was collected and the serum was separated and analyzed immediately. The study protocol was evaluated and approved by the ethics committee of our hospital, and written informed consent was obtained from each subject prior to the evaluation.

2.2. Analysis of C3, C4, and CH50. Serum concentrations of C3 and C4 (the third and fourth components of the complement) were analyzed using kits from Roche Diagnostics (Indianapolis, IN, USA) according to the manufacturer's guidelines. Complement system function (CH50) was evaluated using the liposome immunoassay (Wako Diagnostics, Richmond, VA, USA) according to the manufacturer's guidelines. All values were compared to the normal ranges which were reported as $0.8-1.6 \mathrm{~g} / \mathrm{L}$ for $\mathrm{C} 3,0.1-0.4 \mathrm{~g} / \mathrm{L}$ for $\mathrm{C} 4$, and 23-46 U/mL for CH50.

2.3. Parameters for Disease Severity. The MELD-Na score was used to assess disease severity and calculated as previously described [18]. If the sodium values were below $125 \mathrm{mmol} / \mathrm{L}$, they were set to $125 \mathrm{mmol} / \mathrm{L}$, and if the values were above $140 \mathrm{mmol} / \mathrm{L}$, they were adjusted to $140 \mathrm{mmol} / \mathrm{L}$. Aspartate aminotransferase (AST), alanine aminotransferase (ALT), total bilirubin (Tbil), albumin (ALB), globulin (GLB), and creatinine $(\mathrm{Cr})$ were quantitated using an autoanalyzer (TBA30FR Toshiba, Tokyo, Japan). Prothrombin time (PT) and prothrombin time activity (PTA) were measured using an automatic hemostasis/thrombosis analyzer (STA compact, Holliston, MA, USA). Alpha-fetoprotein (AFP) was determined using Roche's Elecsys system (Hoffmann-La Roche, Basel, Switzerland).

2.4. Virological Assessment. HBV-DNA levels were quantitated by real-time quantitative PCR using the ABI7300 thermocycler (AppliedBiosystems, Foster City, CA, USA). The limit of detection of the assay was 500 copies/mL. Serum HBV markers, including hepatitis B s antigen (HBsAg), hepatitis B $s$ antibody (HBsAb), hepatitis $\mathrm{B}$ e antigen ( $\mathrm{HBeAg}$ ), hepatitis $\mathrm{B}$ e antibody (HBeAb), and hepatitis B c antibody (HBcAb), were determined using the Elecsys system (Hoffmann-La Roche, Basel, Switzerland).

2.5. Statistical Analysis. All data were analyzed using SPSS version 13.0 software (Chicago, IL, USA) and expressed as frequencies, median, and range or as mean \pm standard error. Differences in variables were analyzed using ANOVA and Student's $t$-tests (for normally distributed data) or Kruskal-Wallis and Mann-Whitney $U$ tests (for nonnormally distributed data). Comparisons between C3 grades were performed using the Chi-square test or Kruskal-Wallis test when appropriate for frequencies. Categorical data were analyzed using the Chisquare test and Fisher's exact test. Correlation analysis was evaluated by the Spearman rank correlation test. Survival was analyzed by Kaplan-Meier curve. Multivariate analysis was conducted using backward stepwise Cox proportional hazards regression analysis for the probability of mortality. A two-sided $P<0.05$ was considered statistically significant.

\section{Results}

3.1. Characteristics of Patients. Of a total of 103 consecutive HBV-ACLF patients who met the diagnostic criteria, 


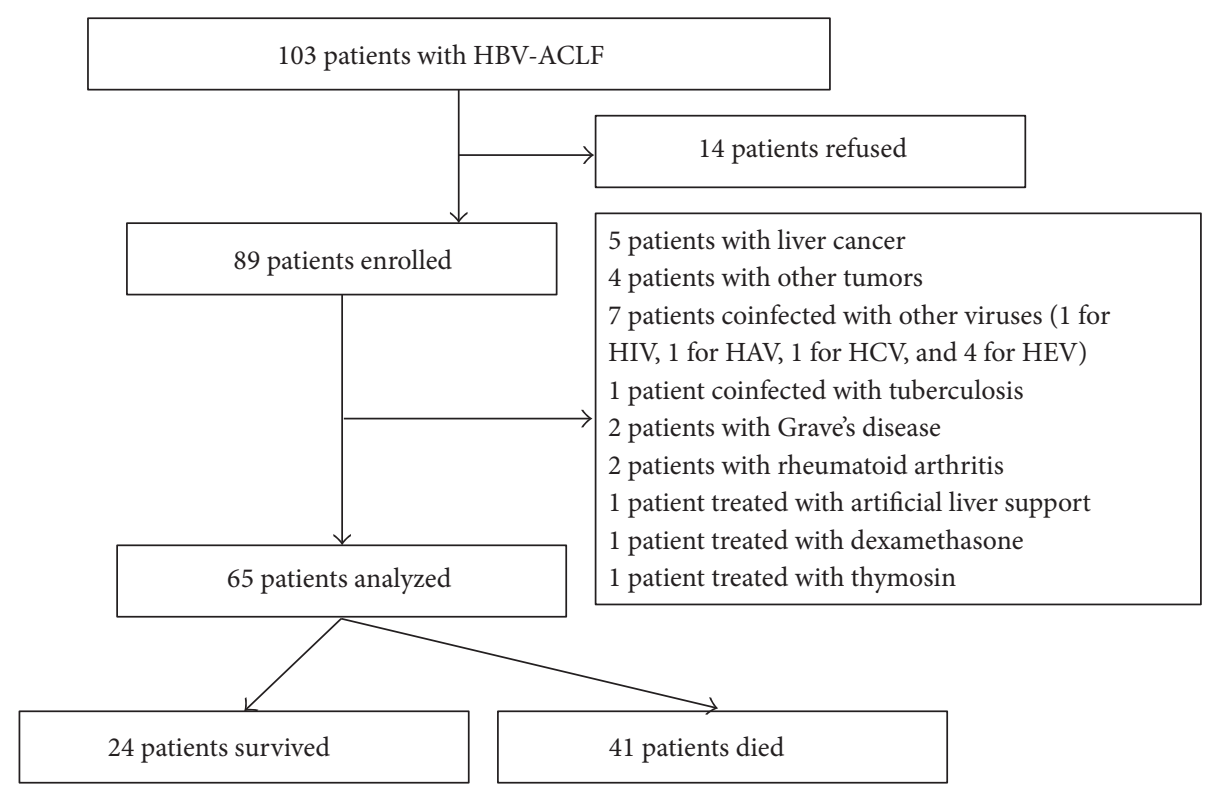

Figure 1: Selection and deposition of patients.

14 patients refused to participate in the study and 24 patients were excluded from the final analysis for the following reasons: 9 patients had tumors (5 patients had liver cancer and 4 had other tumors); 7 patients had coinfection with other viruses; 1 patient was coinfected with tuberculosis; 4 patients had systematic autoimmune diseases ( 2 patients had Grave's disease and 2 had rheumatoid arthritis) and were suspected to have a flare of autoimmune hepatitis; and 3 patients received other medical care (1 received artificial liver support, 1 received dexamethasone, and 1 received thymosin) during their hospital stay. Finally, 65 patients were assigned to the HBV-ACLF group with a MELD-Na score at admission of $31.70 \pm 0.48$. Among them, 55 patients received antiviral therapy due to the presence of HBV-DNA. Our study patients had a median hospital stay of 39 days (range 3-144). Twenty four patients survived and were discharged from the hospital while 41 patients died. A summary of patients' deposition is shown in Figure 1. The characteristics of patients from the HBV-ACLF, CHB, and NC groups are shown in Table 1. No significant differences existed among three groups for the age and gender ratio $(P=0.165$ and $P=0.531$, resp.). Moreover, there were no significant differences in HBeAg levels between the CHB and HBV-ACLF groups $(P=0.529)$.

3.2. C3, C4, and CH50 Levels Were Significantly Lower in HBVACLF Patients Independently of HBeAg Presence. Complement activation is a complicated process and can be triggered by different pathways. Activation leads to convergence of all complement pathways followed by depletion of the central component C3 [4]. Therefore, we first determined the serum C3 levels in HBV-ACLF patients and controls. HBV-ACLF patients had significantly lower serum C3 levels (median $0.384 \mathrm{~g} / \mathrm{L}$ ) compared to $\mathrm{CHB}$ subjects (median $0.795 \mathrm{~g} / \mathrm{L}$, $P<0.001$ ) and normal controls (median $0.970 \mathrm{~g} / \mathrm{L}, P<$ 0.001; Figure 2(a)). Serum C4 and CH50 levels were also significantly lower in HBV-ACLF patients compared to $\mathrm{CHB}$ patients and normal controls (median $0.057 \mathrm{~g} / \mathrm{L}$ versus $0.116 \mathrm{~g} / \mathrm{L}$ versus $0.215 \mathrm{~g} / \mathrm{L}$; median $12 \mathrm{U} / \mathrm{mL}$ versus $35 \mathrm{U} / \mathrm{mL}$ versus $35 \mathrm{U} / \mathrm{mL}$, resp., Figure $2(\mathrm{a}))$. We then determined the correlation between $\mathrm{HBeAg}$ presence and complement expression. In the $\mathrm{CHB}$ group, no significant differences existed in $\mathrm{C} 3, \mathrm{C} 4$, or $\mathrm{CH} 50$ levels between $\mathrm{HBeAg}$-positive $(n=18)$ and HBeAg-negative patients $(n=17)(P=0.373$, $P=0.666$, and $P=0.338$, resp., Figure $2(\mathrm{~b}))$. There was also no significant difference in $\mathrm{C} 3, \mathrm{C} 4$, or $\mathrm{CH} 50$ levels between HBeAg-positive HBV-ACLF patients $(n=28)$ and those who were HBeAg-negative $(n=37)(P=0.420, P=0.740$, and $P=0.474$, resp., Figure 2(c)).

3.3. Lower Complement Levels Indicated Liver Injury and Impaired Liver Regeneration in $\mathrm{HBV}$-ACLF Patients. We analyzed the correlation between serum $\mathrm{C} 3, \mathrm{C} 4$, and $\mathrm{CH} 50$ levels and parameters for disease severity in HBV-ACLF patients. We showed a significant negative correlation between C3 levels and MELD-Na scores $(r=-0.274, P=0.027)$. However, MELD-Na scores were not significantly associated with C4 levels $(r=-0.218, P=0.081)$ or CH50 levels $(r=$ $-0.209, P=0.094$; Figure 3(a)). Interestingly, serum Tbil levels were negatively correlated with $\mathrm{C} 3, \mathrm{C} 4$, and $\mathrm{CH} 50$ levels $(r=-0.277, P=0.005 ; r=-0.285, P=0.004$; and $r=$ $-0.356, P<0.001$, resp., Figure 3(b)), while plasma PTA levels were positively correlated with $\mathrm{C} 3, \mathrm{C} 4$, or $\mathrm{CH} 50$ levels $(r=0.727, P<0.001 ; r=0.575, P<0.001$; and $r=$ $0.636, P<0.001$, resp., Figure 3(c)). There was a significant negative correlation between CH50 levels and HBV-DNA loads $(r=-0.320, P=0.017)$ and a slight negative correlation between C3 levels and HBV-DNA loads $(r=-0.248, P=$ 0.068; Figure 3(d)) in HBV-ACLF patients. We also showed a positive association between serum ALB levels and C3, C4, and CH50 levels $(r=0.444, P<0.001 ; r=0.354, P<0.001$; 
TABLE 1: Clinical characteristics of the populations enrolled in the study.

\begin{tabular}{|c|c|c|c|}
\hline Variables & $\mathrm{NC}(n=16)$ & $\mathrm{CHB}(n=35)$ & $\operatorname{HBV}-\operatorname{ACLF}(n=65)$ \\
\hline Sex (male) & 14 & 32 & 62 \\
\hline Age (years) & $37.69 \pm 2.93$ & $37.69 \pm 1.28$ & $41.52 \pm 1.47$ \\
\hline ALT (U/L) & $23.68 \pm 2.30$ & $529(41-2424)$ & $257(25-1986)$ \\
\hline AST (U/L) & $23.56 \pm 1.84$ & $386(55-2646)$ & $225(78-3023)$ \\
\hline Tbil (mg/dL) & N.D. & $9.50(0.92-49.71)$ & $28.82(10.75-64.82)$ \\
\hline $\mathrm{PT}(\mathrm{s})$ & N.D. & $15.6(12.5-21.2)$ & $28.0(20.2-60.6)$ \\
\hline PTA (\%) & N.D. & $72(46-113)$ & $29(11-40)$ \\
\hline $\operatorname{ALB}(g / L)$ & N.D. & $38.70 \pm 0.69$ & $33.88 \pm 0.66$ \\
\hline $\mathrm{GLB}(\mathrm{g} / \mathrm{L})$ & N.D. & $29.56 \pm 0.87$ & $31.58 \pm 0.90$ \\
\hline $\mathrm{Na}(\mathrm{mmol} / \mathrm{L})$ & N.D. & $139.0(133.8-142.5)$ & $137.2(117.7-146.2)$ \\
\hline Creatinine $(\mu \mathrm{mol} / \mathrm{L})$ & N.D. & $64.0(42-117)$ & $63.4(37.5-267)$ \\
\hline $\operatorname{AFP}(n g / m L)$ & N.D. & $108.0(1.7-1000)$ & $53.5(2.8-1112)$ \\
\hline DNA $\left(\log _{10} \mathrm{IU} / \mathrm{mL}\right)$ & N.D. & $5.69 \pm 0.24$ & $5.58 \pm 0.20^{*}$ \\
\hline HBsAg-positive & 0 & 35 & 65 \\
\hline HBsAb-positive & 16 & 0 & 0 \\
\hline HBeAg-positive & 0 & 18 & 28 \\
\hline $\mathrm{HBeAb}$-positive & 0 & 17 & 37 \\
\hline HBcAb-positive & 0 & 35 & 65 \\
\hline
\end{tabular}

Data are shown as means and standard error or median and range. ACLF, acute-on-chronic liver failure; CHB, chronic hepatitis B; NC, normal control; ALT, alanine aminotransferase; AST, aspartate aminotransferase; PT, prothrombin time; ALB, albumin; GLB, globulin; AFP, alpha-fetoprotein; and N.D., not determined. ${ }^{*} \mathrm{HBV}$-DNA could not be detected in 10 patients with HBV-ACLF.

and $r=0.363, P<0.001$, resp., Figure 4(a)) in these HBVinfected subjects. Furthermore, there was a positive correlation between serum C3 levels and AFP levels $(r=0.283$, $P=0.022$; Figure 4(b)) in HBV-ACLF patients. However, no significant correlations existed between $\mathrm{C} 3, \mathrm{C} 4$, or $\mathrm{CH} 50$ levels and ALT levels, AST levels, or GLB levels in these HBVinfected patients (data not shown). These findings suggest that lower complement levels were associated with liver injury and impaired liver regeneration in HBV-ACLF patients.

3.4. Survival Rate Varied Depending on C3 Levels. We examined the correlation between clinical outcome and expression of C3, C4, and CH50. Only C3 levels were significantly lower in HBV-ACLF patients who died (median $0.319 \mathrm{~g} / \mathrm{L}, n=$ 41) compared to patients who survived (median $0.472 \mathrm{~g} / \mathrm{L}$, $n=25, P=0.003$; Figure 5(a)). There was no significant difference in $\mathrm{C} 4$ or $\mathrm{CH} 50$ levels between patients who died and those who survived $(P=0.901$ and $P=0.335$, resp., Figures 5(b) and 5(c)). Based on further analysis, we grouped the 65 patients into 3 groups based on C3 values (low grade: $0.16-0.30 \mathrm{~g} / \mathrm{L}, n=22$; moderate grade: $0.3-0.45 \mathrm{~g} / \mathrm{L}, n=21$; and high grade: $0.45-1.27 \mathrm{~g} / \mathrm{L}, n=22$ ). The minimum C3 was $0.16 \mathrm{~g} / \mathrm{L}$, 33th percentile was $0.30 \mathrm{~g} / \mathrm{L}$, 66 th percentile was $0.45 \mathrm{~g} / \mathrm{L}$, and maximum was $1.27 \mathrm{~g} / \mathrm{L}$. In a median hospital stay of 39 days (range 3-144), 41 patients (63\%) died during the hospital stay of which 19 patients $(86 \%)$ had low grade C3, 13 patients had (62\%) moderate grade C3, and 9 patients (41\%) had high grade C3, making a significant difference $(P=$ 0.008 ). A majority of deaths occurred due to the development of multiorgan failure caused by progressive liver failure, leading to hepatorenal syndrome and hepatic encephalopathy. In addition, using the Kaplan-Meier analysis, the log-rank test revealed a significant difference in C3 levels among three groups. Patients with a high grade of C3 had a significantly higher survival rate compared to those with moderate grade or low grade (log-rank 15.443, $P<0.001$, Figure $5(\mathrm{~d})$ ).

3.5. C3 Was an Independent Predictor of Mortality. We analyzed the baseline clinical and laboratory variables as possible predictors of mortality. Using univariate Cox regression analysis, we showed that age, MELD-Na score, PTA, AST, $\mathrm{Cr}$, and $\mathrm{C} 3$ grade were significantly associated with mortality (Table 2). Using these significant variables in multivariate Cox regression analysis, we showed that MELD-Na score (HR 1.189, 95\% CI 1.071-1.320, $P=0.001$ ), Cr (HR 1.013, 95\% CI $1.001-1.025, P=0.032$ ), and C3 grade (low grade: HR 2.771, 95\% CI 1.191-6.446, $P=0.018$ and moderate grade: HR 3.071, 95\% CI 1.201-7.852, $P=0.019$ ) were independent baseline predictors of mortality in HBV-ACLF patients.

\section{Discussion}

Although the role of complement has been explored extensively in several liver diseases, surprisingly few reports have examined its role in HBV-ACLF patients. To our knowledge, ours is the first study which extensively explores the role of complement in a large sample cohort of HBV-ACLF patients. We present evidence that (1) C3, C4, and CH50 levels were significantly lower in HBV-ACLF patients, (2) lower complement levels were closely correlated with liver injury and impaired liver regeneration, and (3) mortality varied depending on C3 levels and C3 levels may be an independent predictor of mortality. Collectively, our data support the idea 

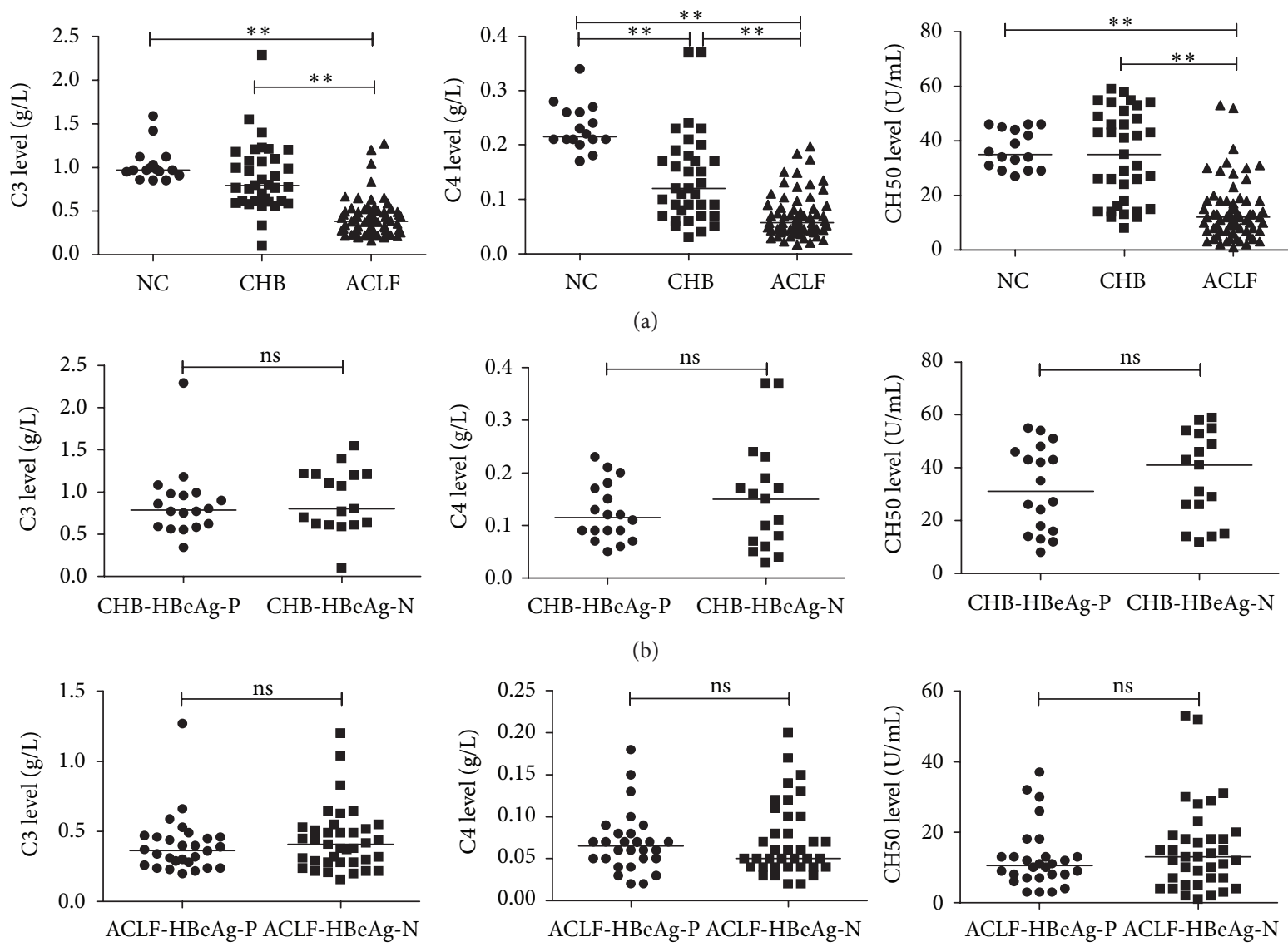

(c)

Figure 2: C3, C4, and CH50 levels were significantly lower in HBV-ACLF patients, independently of HBeAg presence. Pooled data indicated the levels of $\mathrm{C} 3, \mathrm{C} 4$, and $\mathrm{CH} 50$ in each group, where the lines indicated the median. (a) Serum C3, C4, and CH50 levels were significantly lower in HBV-ACLF patients compared to CHB subjects and NC group. No significant differences existed between patients with HBeAg positive and those with HBeAg negative, neither in CHB group (b) nor in HBV-ACLF group (c). ${ }^{* *} P<0.01$; ns, not significant. ACLF, acute-on-chronic liver failure; $\mathrm{CHB}$, chronic hepatitis B; NC, normal control; HBeAg-P, HBeAg-positive; HBeAg-N, HBeAg-negative.

that complement plays a key role in the pathogenesis of HBVACLF and C3 may be a potential target for developing pharmaceuticals that interrupt or dampen complement mediated liver injury.

Accumulating data have indicated a pathogenic role of complement in a variety of liver diseases [8-13]. However, the role of the complement in HBV-ACLF patients is poorly understood. Immunohistochemical analysis has shown the deposition of the membrane attack complex around necrotic areas in patients with fulminant hepatitis, which indicates activation of the complement system and contributes to liver injury [19]. In a mouse model of fulminant hepatic failure, C3-/- mice survived longer and displayed reduced liver inflammation and attenuated pathological damage compared with wild-type mice [14]. The complement was also found to be deposited in the liver parenchyma of two HBV-ACLF patients [15]. These studies led us to hypothesize that complement may mediate hepatic injury in liver failure. Our data suggest that complement system was closely correlated with liver injury in HBV-ACLF patients. There are several lines of evidence to support this notion. Firstly, we demonstrated that HBV-ACLF patients had lower serum C3, C4, and CH50 levels compared to $\mathrm{CHB}$ patients or healthy individuals. Secondly, C3 levels were negatively correlated with MELD-Na score, which was an important predictor of clinical outcome in HBV-ACLF [18]. Thirdly, serum C3, C4, and CH50 levels in $\mathrm{HBV}$-infected patients were negatively associated with serum Tbil levels but positively correlated with plasma PTA levels, which often serve as markers of liver injury [20]. These results suggest that complement was closely associated with HBVinfection induced liver damage, which may contribute to the pathogenesis of HBV-ACLF.

In addition to its pathogenic role in hepatic injury, recent evidence indicated that complement was required for liver regeneration [21-23]. Using a mouse model of 70\% partial hepatectomy, mice deficient in C3 and C5 exhibited impaired liver regeneration and high mortality after liver resection. However, reconstitution of the complement-deficient mice with $\mathrm{C} 3 \mathrm{a}$ or $\mathrm{C} 5 \mathrm{a}$ improved the regenerative response [22]. Complement activation products $\mathrm{C} 3 \mathrm{a}$ and $\mathrm{C} 5 \mathrm{a}$ were also shown to play a key role in the proliferative response and hepatocyte regeneration via the TNF- $\alpha$ and IL-6 pathways [22]. 

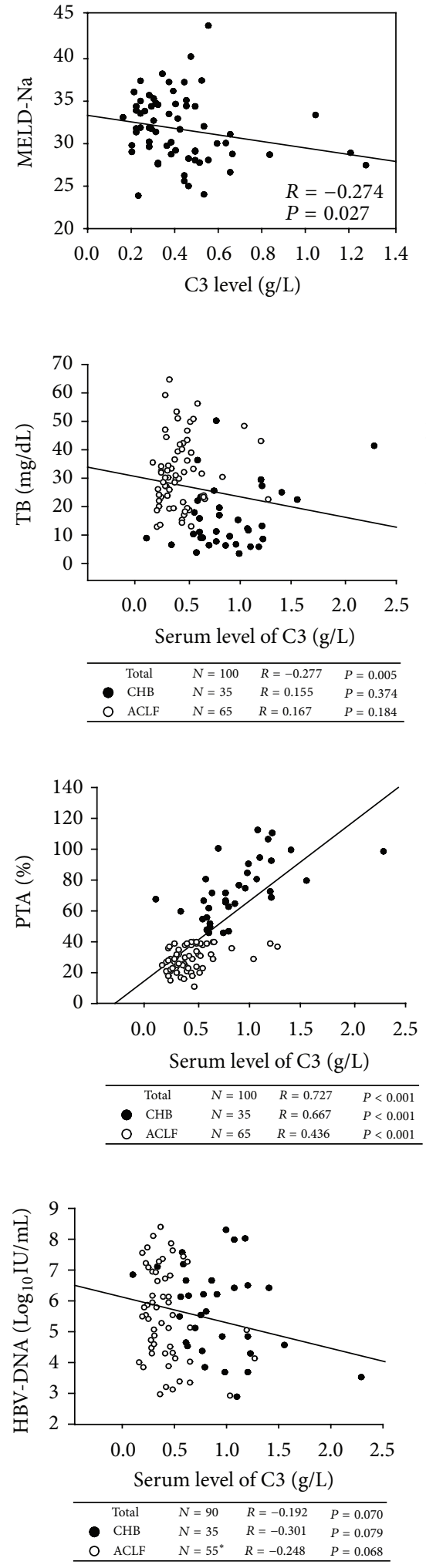

FIGURE 3: Lower complement levels indicated liver injury in HBV-ACLF patients. A significant negative correlation was found between C3 levels and MELD-Na scores (a). C3, C4, and CH50 levels in serum were negatively correlated with serum Tbil levels (b) but positively associated with plasma PTA levels (c) in HBV-infected patients (chronic hepatitis B (CHB) and acute-on-chronic liver failure (ACLF)). There was a significant negative correlation between CH50 levels and HBV-DNA loads and a slight negative correlation between C3 levels and HBV-DNA loads in HBV-ACLF patients (d). Solid line, linear growth trend; $R$, correlation coefficient. $P$ values were shown. 

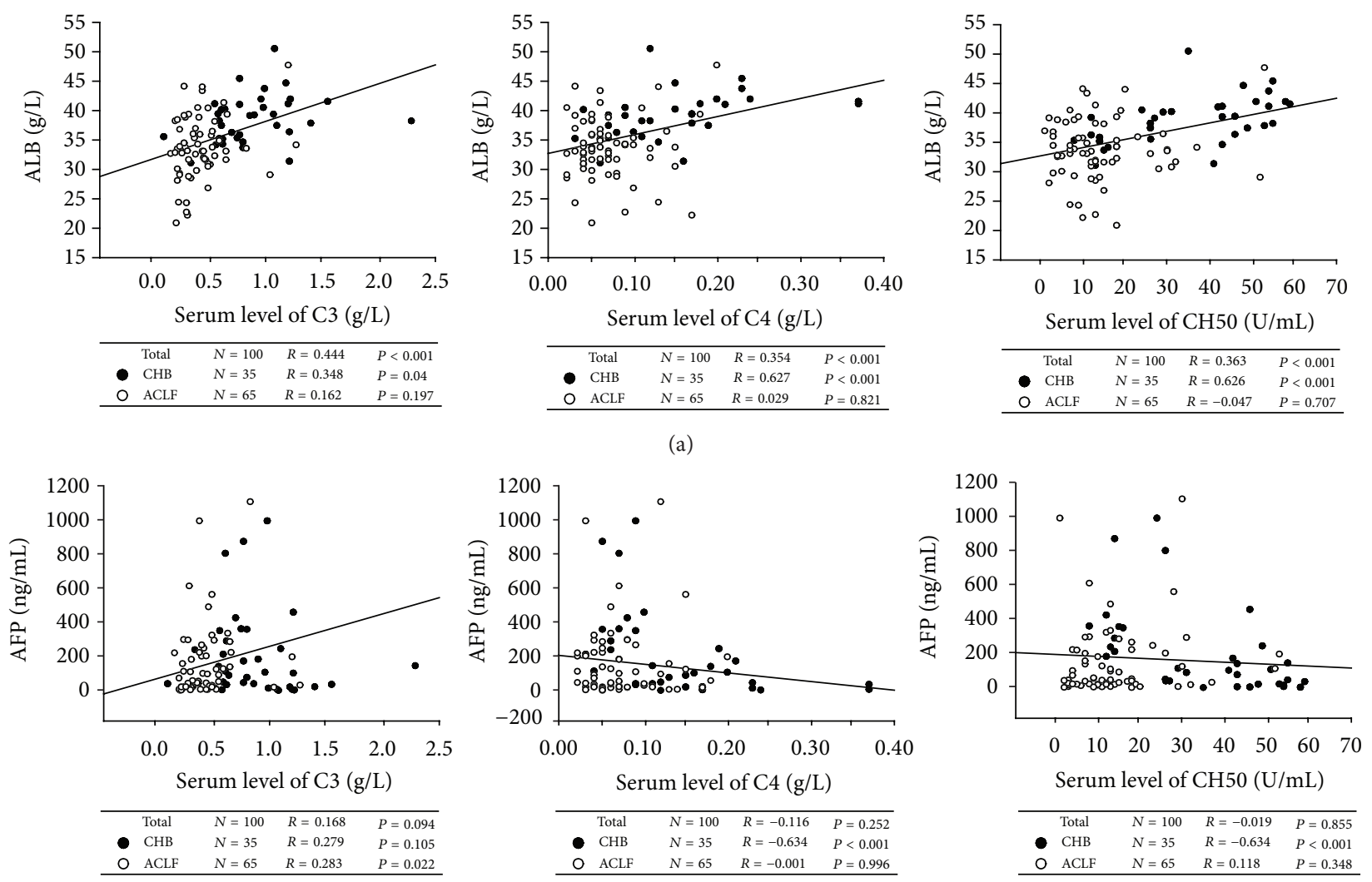

(b)

FIGURE 4: Lower complement levels indicated impaired liver regeneration in HBV-ACLF patients. (a) C3, C4, and CH50 levels in serum were positively associated with serum ALB levels in HBV-infected patients (chronic hepatitis B (CHB) and acute-on-chronic liver failure (ACLF)). (b) A positive correlation was found between serum C3 levels and AFP levels in HBV-ACLF patients. Solid line, linear growth trend; $R$, correlation coefficient. $P$ values were shown.

TABLE 2: Factors associated with mortality in univariate Cox regression analysis.

\begin{tabular}{lccc}
\hline Variables & $P$ value & Hazard ratio & $95 \%$ CI \\
\hline Age (years) & 0.032 & 1.029 & $1.002-1.057$ \\
HBeAg-positive & 0.190 & 1.538 & $0.807-2.931$ \\
MELD-Na & $<0.001$ & 1.206 & $1.100-1.322$ \\
Tbil (mg/dL) & 0.490 & 1.009 & $0.984-1.034$ \\
PTA (\%) & $<0.001$ & 0.919 & $0.880-0.959$ \\
AST (U/L) & 0.019 & 1.001 & $1.000-1.001$ \\
ALT (U/L) & 0.242 & 1.000 & $1.000-1.001$ \\
ALB $(\mathrm{g} / \mathrm{L})$ & 0.631 & 1.014 & $0.958-1.074$ \\
GLB $(\mathrm{g} / \mathrm{L})$ & 0.912 & 1.002 & $0.964-1.042$ \\
Creatinine $(\mu \mathrm{mol} / \mathrm{L})$ & 0.002 & 1.017 & $1.006-1.028$ \\
AFP $(\mathrm{ng} / \mathrm{mL})$ & 0.753 & 1.000 & $0.998-1.001$ \\
DNA $(\mathrm{log}$ 10 $\mathrm{IU} / \mathrm{mL})$ & 0.061 & 1.004 & $1.000-1.009$ \\
C3 $(\mathrm{g} / \mathrm{L})$ & 0.001 & & \\
$0.16 \leq \mathrm{C} 3<0.3$ & $<0.001$ & 4.625 & $2.018-10.600$ \\
$0.3 \leq \mathrm{C} 3<0.45$ & 0.007 & 3.483 & $1.402-8.653$ \\
C4 $(\mathrm{g} / \mathrm{L})$ & 0.816 & 2.566 & $0.001-7060.782$ \\
CH50 $(\mathrm{U} / \mathrm{mL})$ & 0.742 & 0.994 & $0.960-1.030$ \\
Antiviral therapy & 0.085 & 1.811 & $0.921-3.557$ \\
\hline
\end{tabular}

AST, aspartate aminotransferase; ALT, alanine aminotransferase; ALB, albumin; GLB, globulin; and AFP, alpha-fetoprotein.
A similar role for complement and for $\mathrm{C} 3 \mathrm{a}$ receptor $(\mathrm{C} 3 \mathrm{aR})$ and $\mathrm{C} 5 \mathrm{aR}$ signaling in liver regeneration has been demonstrated in a mouse model of CCL4-induced liver toxicity [21, 23]. Our data were consistent with these studies and showed that complement was necessary for liver regeneration. We showed a positive correlation between $\mathrm{C} 3, \mathrm{C} 4$, and $\mathrm{CH} 50$ levels and serum ALB levels and also a positive correlation between serum C3 levels and serum AFP, which is considered to be a valuable marker of hepatocyte regeneration. Taken together, these results suggest that complement plays a crucial role in impairment of liver regeneration. It is important to further study the role of complement in liver regeneration in patients with HBV-ACLF.

A number of studies have suggested that reactivation of HBV infection may be an initial event in inducing the pathogenesis of HBV-ACLF subjects [24-26]. Reactivation is often spontaneous but can also be triggered by cancer chemotherapy, immune suppression, or alteration in immune function. A previous study showed that spontaneous acute exacerbation of $\mathrm{CHB}$ infection was seen with a cumulative probability of $15 \%-37 \%$ after 4 years of follow-up [27]. As a result, APASL guidelines strongly recommend initiation of antiviral therapy in HBV-ACLF patients [28]. A recent metaanalysis showing that nucleoside analogues can significantly 


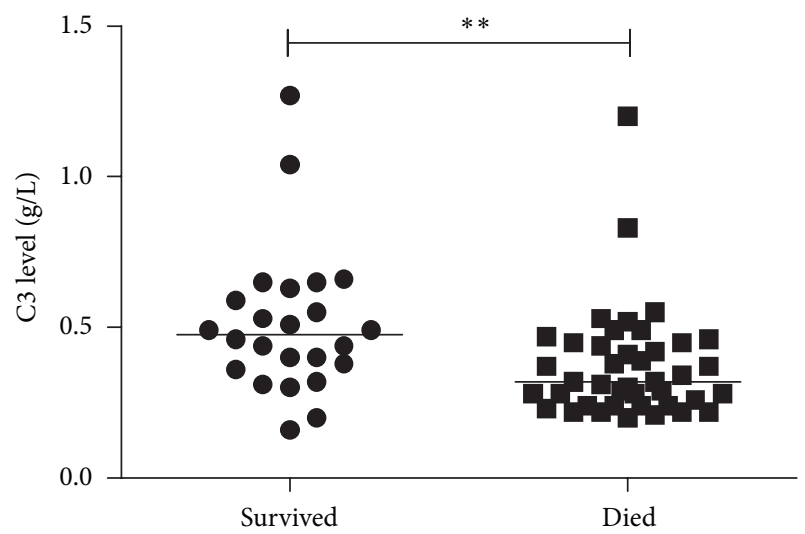

(a)

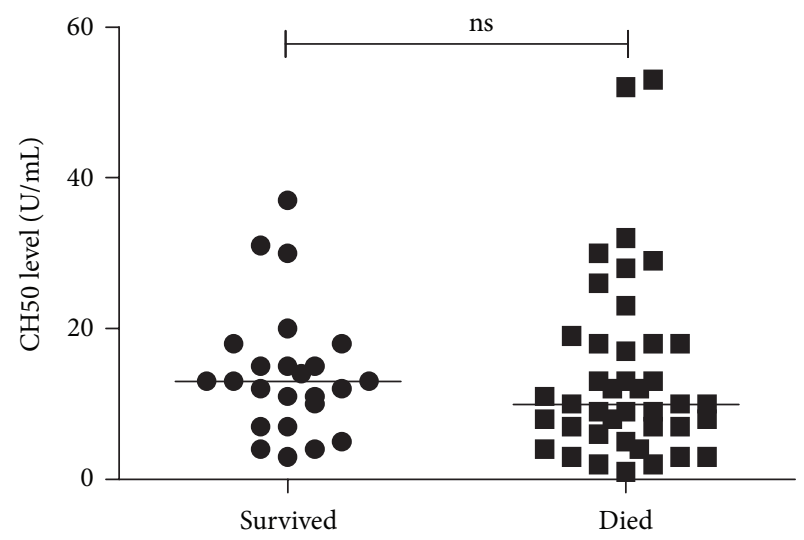

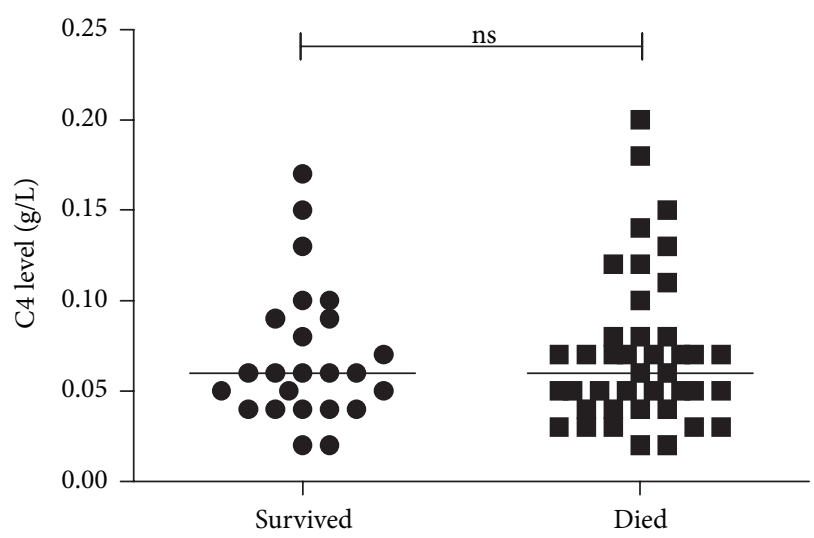

(b)

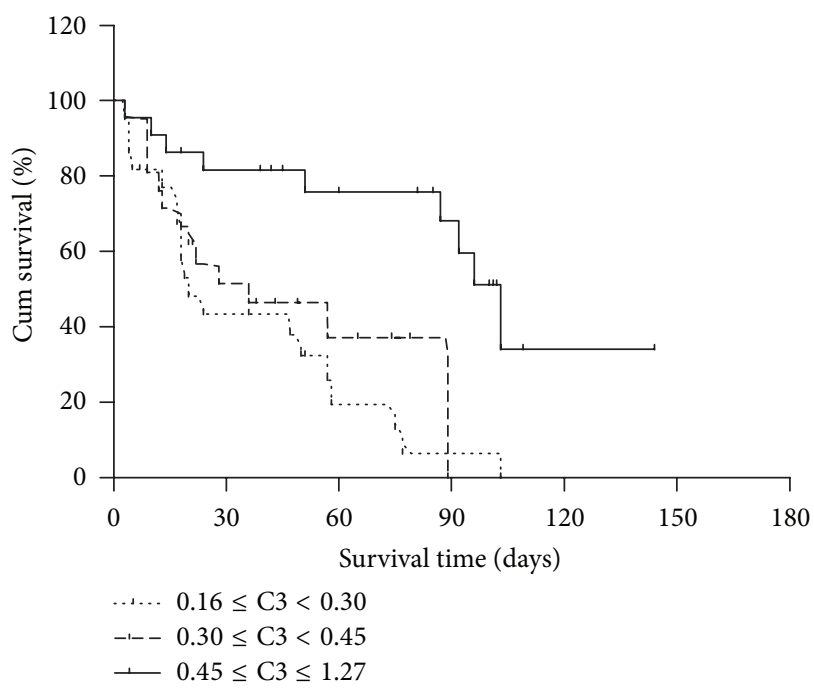

(d)

Figure 5: Survival rate varied depending on C3 levels. Pooled data indicated the levels of C3, C4, and CH50 in patients who died ( $n=41)$ and those who survived $(n=25)$, where the lines indicated the median. Serum C3 levels were significantly lower in HBV-ACLF patients who died compared to patients who survived (a). However, no significant differences in serum C4 or CH50 levels were found between them (b and c). (d) Using the Kaplan-Meier analysis, the log-rank test revealed that a significant difference existed among three groups according to C3 levels, and patients with a high grade of C3 had a significantly higher survival rate compared to those with moderate grade or low grade (log-rank 15.443, $P<0.001) .{ }^{* *} P<0.01$; ns, not significant.

improve the survival of HBV-ACLF patients [29] highlighted the role of HBV replication in HBV-ACLF. In our study, CH50 levels and C3 levels were negatively correlated with HBVDNA loads at admission prior to receiving antiviral therapy. The data indicated that HBV replication, at least in part, may participate in the complement mediated response and may be a contributor to liver injury. However, it will be important to further study the direct interaction between HBV replication and complement mediated inflammation.

Although antiviral therapy using nucleoside analogues is an important aspect of treatment aimed at inhibiting HBVDNA replication, the outcome of HBV-ACLF patients is still extremely poor. In our study, $55 \mathrm{HBV}$-ACLF patients with detectable HBV-DNA were treated with antiviral therapy. However, the mortality in this cohort was as high as $63 \%$ which was consistent with data from another report [1]. These data emphasize the limitations of current comprehensive care and underscore the importance of developing novel treatment strategies. Previous studies using different animal models showed that $\mathrm{C} 3$ plays a pathogenic role in various liver diseases. C3 deficiency or $\mathrm{C} 3 \mathrm{a}$ antagonists improved survival in alcoholic liver disease or in liver failure models [11, 14]. Interestingly, we showed that mortality was significantly lower in patients with a high grade of $\mathrm{C} 3$ compared to patients with moderate grade, while patients with a low C3 grade had the highest mortality. In addition, we showed that C3 levels at admission were an independent risk factor of mortality. Taken together, these data emphasize the importance of $\mathrm{C} 3$ in the pathogenesis of HBV-ACLF and suggest that it could be a target for immunotherapy intervention.

This study has two important limitations. Firstly, we did not analyze the complement levels in the microenvironment of liver tissues in HBV-ACLF patients due to poor coagulation, which may be a contraindication for liver biopsy. It 
is important to further investigate the relationship between the microenvironment of liver tissues and the circulating complement levels. Secondly, this is a descriptive and observational study showing that complement system was involved in the pathogenesis of HBV-ACLF and C3 levels at admission were a predictor of mortality in HBV-ACLF patients. Future studies to explore the mechanisms underlying the role of complement in HBV-ACLF are urgently needed.

In summary, we showed that (1) complement components were significantly decreased in HBV-ACLF patients, suggesting that the complement may be implicated in the pathogenesis of HBV-ACLF; (2) lower complement was closely correlated with liver injury and impaired liver regeneration; and (3) the survival rate varied depending on C3 levels and C3 levels at admission were an independent risk factor of mortality in HBV-ACLF patients. Our findings shed new light on the pathogenic mechanisms involved in pathogenesis of $\mathrm{HBV}$-ACLF and an approach of modulated C3 represents a potential therapeutic strategy for patients with HBV-ACLF.

\author{
Abbreviations \\ ACLF: Acute-on-chronic liver failure \\ CHB: Chronic hepatitis B \\ NC: Normal control \\ HBV: Hepatitis B virus \\ C3: The third complement component \\ C4: The fourth complement component \\ PTA: Prothrombin time activity \\ Tbil: Total bilirubin.
}

\section{Competing Interests}

The authors declare that they have no competing interests.

\section{Authors' Contributions}

Geng-lin Zhang and Ting Zhang contributed equally to this work.

\section{Acknowledgments}

This study was supported by grants from the National Science and Technology Major Project (2016ZX10002008, 2016ZX10002009, and 2016ZX10002010), Guangzhou Science and Technology Project (1561000155), and the 111 Project (B12003).

\section{References}

[1] R. Jalan, P. Gines, J. C. Olson et al., "Acute-on chronic liver failure," Journal of Hepatology, vol. 57, no. 6, pp. 1336-1348, 2012.

[2] F.-S. Wang and Z. Zhang, "How can acute-on-chronic liver failure be accurately identified?" Nature Reviews Gastroenterology \& Hepatology, vol. 10, no. 7, pp. 390-391, 2013.

[3] H. E. Wasmuth, D. Kunz, E. Yagmur et al., "Patients with acute on chronic liver failure display 'sepsis-like' immune paralysis," Journal of Hepatology, vol. 42, no. 2, pp. 195-201, 2005.
[4] X. Qin and B. Gao, "The complement system in liver diseases," Cellular \& Molecular Immunology, vol. 3, no. 5, pp. 333-340, 2006.

[5] P. Gasque, "Complement: a unique innate immune sensor for danger signals," Molecular Immunology, vol. 41, no. 11, pp. 10891098, 2004.

[6] M. J. Walport, "Complement. Second of two parts," The New England Journal of Medicine, vol. 344, no. 15, pp. 1140-1144, 2001.

[7] P. A. Ward, "The dark side of C5a in sepsis," Nature Reviews Immunology, vol. 4, no. 2, pp. 133-142, 2004.

[8] A. Clark, A. Weymann, E. Hartman et al., "Evidence for nontraditional activation of complement factor $\mathrm{C} 3$ during murine liver regeneration," Molecular Immunology, vol. 45, no. 11, pp. $3125-3132,2008$.

[9] S. Hillebrandt, H. E. Wasmuth, R. Weiskirchen et al., "Complement factor 5 is a quantitative trait gene that modifies liver fibrogenesis in mice and humans," Nature Genetics, vol. 37, no. 8, pp. 835-843, 2005.

[10] S. He, C. Atkinson, F. Qiao, K. Cianflone, X. Chen, and S. Tomlinson, "A complement-dependent balance between hepatic ischemia/reperfusion injury and liver regeneration in mice," Journal of Clinical Investigation, vol. 119, no. 8, pp. 2304-2316, 2009.

[11] B. Gao, E. Seki, D. A. Brenner et al., "Innate immunity in alcoholic liver disease," American Journal of Physiology-Gastrointestinal and Liver Physiology, vol. 300, no. 4, pp. G516-G525, 2011.

[12] S. S. Rensen, Y. Slaats, A. Driessen et al., "Activation of the complement system in human nonalcoholic fatty liver disease," Hepatology, vol. 50, no. 6, pp. 1809-1817, 2009.

[13] B. Mazumdar, H. Kim, K. Meyer et al., "Hepatitis C virus proteins inhibit C3 complement production," Journal of Virology, vol. 86, no. 4, pp. 2221-2228, 2012.

[14] S. Sun, Y. Guo, G. Zhao et al., "Complement and the alternative pathway play an important role in LPS/D-GalN-induced fulminant hepatic failure," PloS ONE, vol. 6, no. 11, Article ID e26838, 2011.

[15] P. Farci, G. Diaz, Z. Chen et al., "B cell gene signature with massive intrahepatic production of antibodies to hepatitis $B$ core antigen in hepatitis B virus-associated acute liver failure," Proceedings of the National Academy of Sciences of the United States of America, vol. 107, no. 19, pp. 8766-8771, 2010.

[16] G.-L. Zhang, D.-Y. Xie, B.-L. Lin et al., "Imbalance of interleukin-17-producing CD4 T cells/regulatory T cells axis occurs in remission stage of patients with hepatitis B virus-related acute-on-chronic liver failure," Journal of Gastroenterology and Hepatology, vol. 28, no. 3, pp. 513-521, 2013.

[17] L. Peng, D.-Y. Xie, B.-L. Lin et al., "Autologous bone marrow mesenchymal stem cell transplantation in liver failure patients caused by hepatitis B: short-term and long-term outcomes," Hepatology, vol. 54, no. 3, pp. 820-828, 2011.

[18] A. E. Ruf, W. K. Kremers, L. L. Chavez, V. I. Descalzi, L. G. Podesta, and F. G. Villamil, "Addition of serum sodium into the MELD score predicts waiting list mortality better than MELD alone," Liver Transplantation, vol. 11, no. 3, pp. 336-343, 2005.

[19] B.-N. Pham, J.-F. Mosnier, F. Durand et al., "Immunostaining for membrane attack complex of complement is related to cell necrosis in fulminant and acute hepatitis," Gastroenterology, vol. 108, no. 2, pp. 495-504, 1995.

[20] B. Rehermann and M. Nascimbeni, "Immunology of hepatitis B virus and hepatitis $C$ virus infection," Nature Reviews Immunology, vol. 5, no. 3, pp. 215-229, 2005. 
[21] M. M. Markiewski, D. Mastellos, R. Tudoran et al., "C3a and C3b activation products of the third component of complement (C3) are critical for normal liver recovery after toxic injury," Journal of Immunology, vol. 173, no. 2, pp. 747-754, 2004.

[22] C. W. Strey, M. Markiewski, D. Mastellos et al., "The proinflammatory mediators $\mathrm{C} 3 \mathrm{a}$ and $\mathrm{C} 5 \mathrm{a}$ are essential for liver regeneration," The Journal of Experimental Medicine, vol. 198, no. 6, pp. 913-923, 2003.

[23] D. Mastellos, J. C. Papadimitriou, S. Franchini, P. A. Tsonis, and J. D. Lambris, "A novel role of complement: mice deficient in the fifth component of complement (C5) exhibit impaired liver regeneration," Journal of Immunology, vol. 166, no. 4, pp. 24792486, 2001.

[24] H. E. Kohrt, D. L. Ouyang, and E. B. Keeffe, "Antiviral prophylaxis for chemotherapy-induced reactivation of chronic hepatitis B virus infection," Clinics in Liver Disease, vol. 11, no. 4, pp. 965-991, 2007.

[25] W.-H. Sheng, J.-H. Kao, P.-J. Chen et al., "Evolution of hepatitis B serological markers in HIV-infected patients receiving highly active antiretroviral therapy," Clinical Infectious Diseases, vol. 45, no. 9, pp. 1221-1229, 2007.

[26] H. J. Flink, D. Sprengers, B. E. Hansen et al., "Flares in chronic hepatitis B patients induced by the host or the virus? Relation to treatment response during Peg-interferon $\alpha$-2b therapy," Gut, vol. 54, no. 11, pp. 1604-1609, 2005.

[27] A. S. F. Lok and C.-L. Lai, "Acute exacerbations in Chinese patients with chronic hepatitis B virus (HBV) infection. Incidence, predisposing factors and etiology," Journal of Hepatology, vol. 10, no. 1, pp. 29-34, 1990.

[28] S. K. Sarin, A. Kumar, J. A. Almeida et al., "Acute-on-chronic liver failure; consensus recommendations of the Asian Pacific Association for the Study of the Liver (APASL)," Hepatology International, vol. 3, no. 1, pp. 269-282, 2009.

[29] F. Xie, L. Yan, J. Lu et al., "Effects of nucleoside analogue on patients with chronic hepatitis B-associated liver failure: metaanalysis," PLoS ONE, vol. 8, no. 1, Article ID e54773, 2013. 


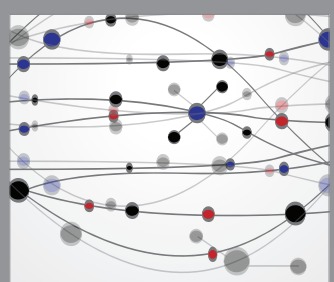

The Scientific World Journal
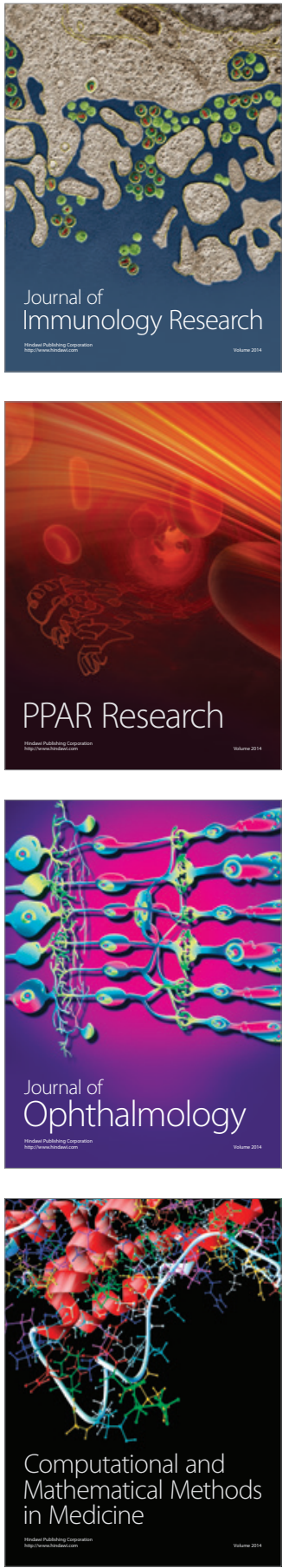

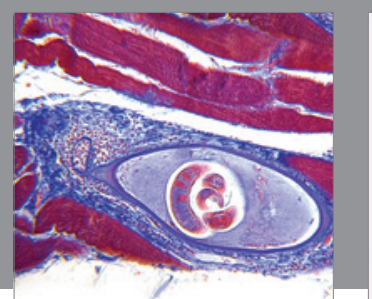

Gastroenterology Research and Practice

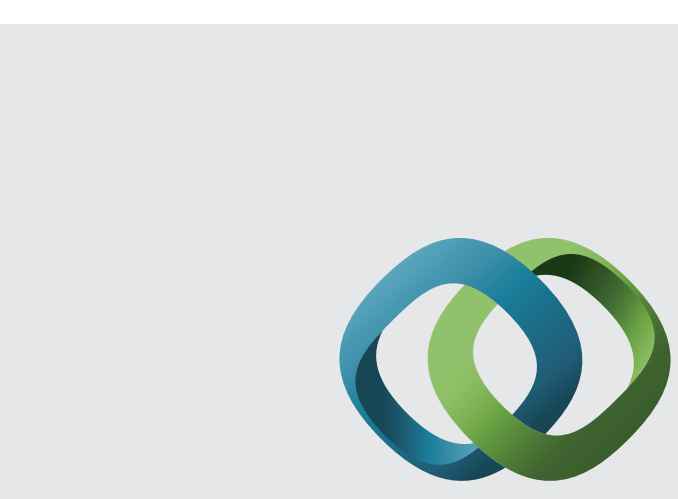

\section{Hindawi}

Submit your manuscripts at

http://www.hindawi.com
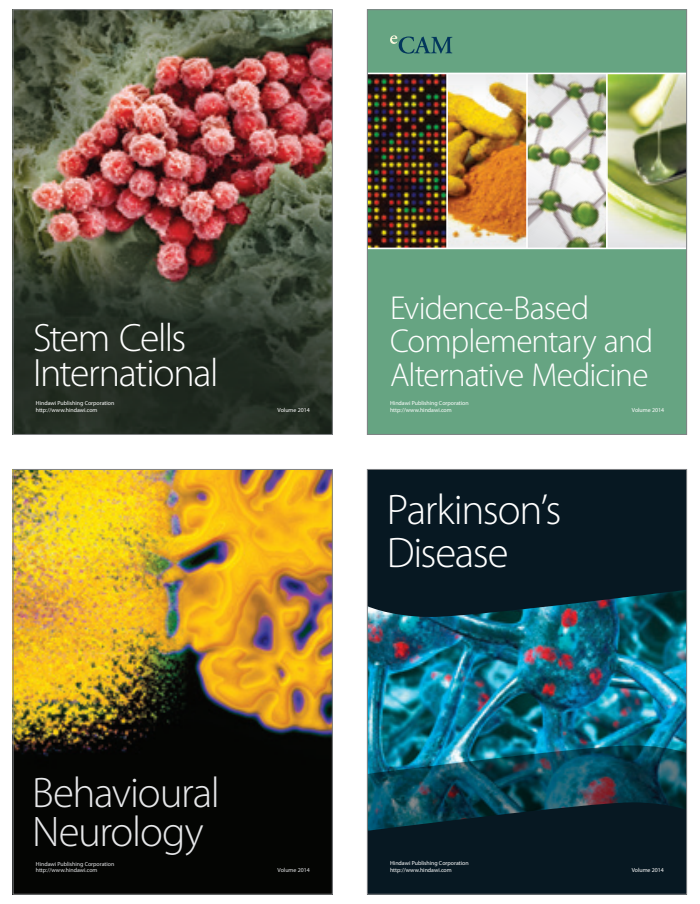
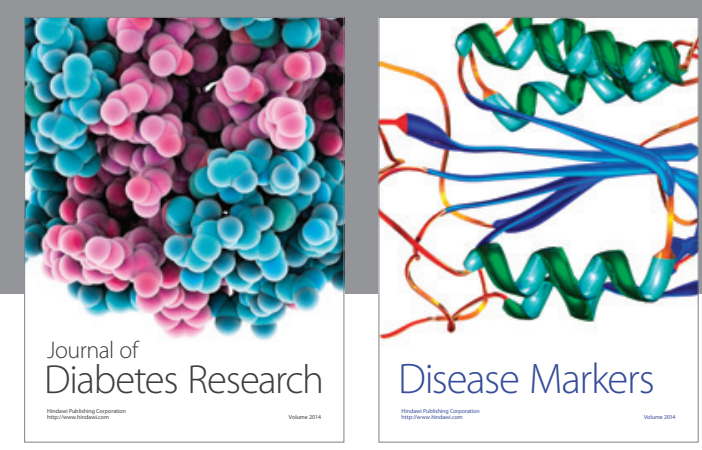

Disease Markers
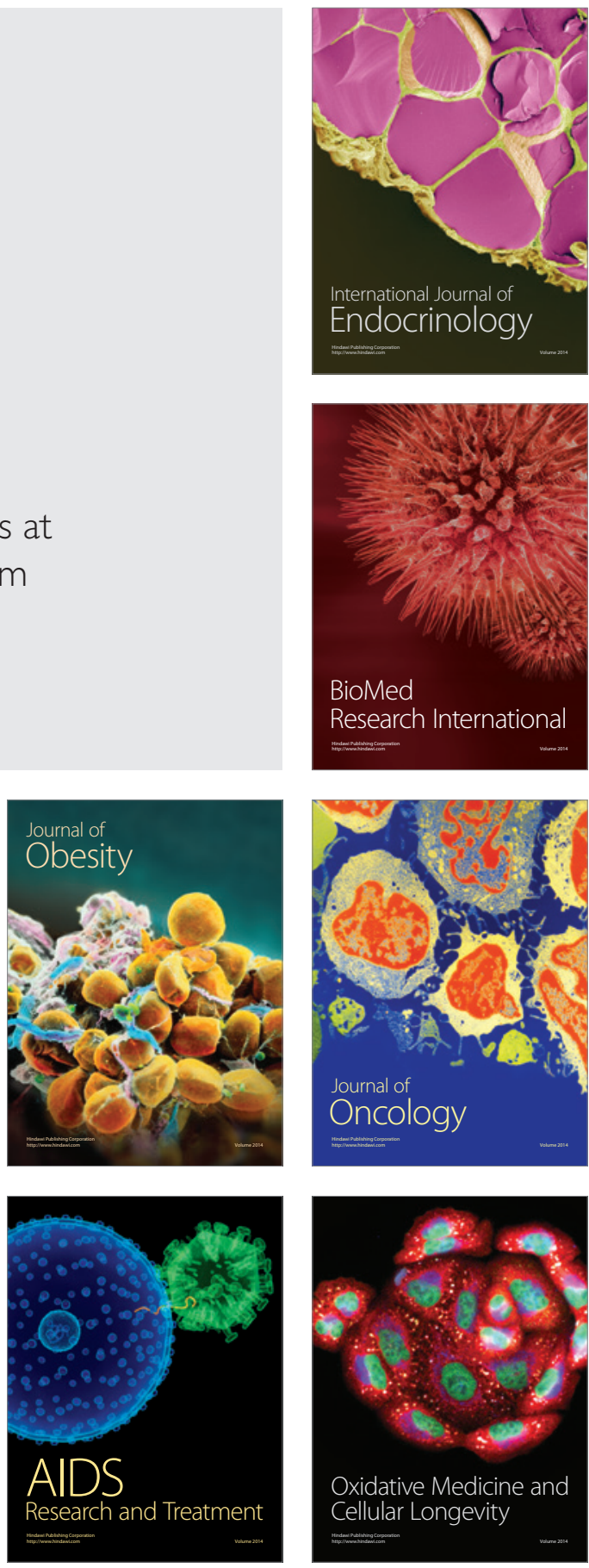